OPEN ACCESS

Edited by:

Daniela Smirni,

University of Palermo, Italy

Reviewed by:

Carmen Berenguer,

University of Valencia, Spain

Marie Geurten,

University of Liège, Belgium

${ }^{*}$ Correspondence:

Andreia Veloso

andreiaveloso@fpce.up.pt

${ }^{\dagger}$ These authors share last authorship

Specialty section:

This article was submitted to Neuropsychology,

a section of the journal

Frontiers in Psychology

Received: 24 September 2019

Accepted: 16 December 2019

Published: 14 January 2020

Citation:

Veloso A, Vicente SG and Filipe MG

(2020) Effectiveness of Cognitive

Training for School-Aged Children and

Adolescents With Attention

Deficit/Hyperactivity Disorder: A

Systematic Review.

Front. Psychol. 10:2983.

doi: 10.3389/fpsyg.2019.02983

\section{Effectiveness of Cognitive Training for School-Aged Children and Adolescents With Attention Deficit/Hyperactivity Disorder: A Systematic Review}

\author{
Andreia Veloso*, Selene G. Vicente ${ }^{\dagger}$ and Marisa G. Filipe ${ }^{\dagger}$ \\ Faculty of Psychology and Education Sciences, Centre for Psychology, University of Porto, Porto, Portugal
}

Problems with executive functions (EF) are hallmark characteristics of Attention Deficit/Hyperactivity Disorder (ADHD). Therefore, this review analyzed the efficacy of cognitive training for EF in reducing ADHD symptomatology and improving educational, interpersonal, and occupational outcomes in children and adolescents with this disorder. A systematic search, using a PICO (population/participant, intervention/indicator, comparator/control, outcome) framework was carried out. From 2008 to 2018, resorting to EBSCOhost, the following databases were searched: Academic Search Complete, ERIC, MEDLINE with Full Text, PsycARTICLES, PsycINFO, and Psychology and Behavioral Sciences Collection. Twenty-two studies were included in this review. Of the 18 studies that reported performance-based measures of EF, 13 found improvements and five did not. Overall, 17 studies showed positive transfer effects on ADHD symptomatology, EF, academic improvement, reduced off-task behavior, and/or enhanced social skills. Of the nine studies that performed follow-up sessions, seven concluded that the treatment effects were maintained over time. In sum, results showed that cognitive training can be an effective intervention for children and adolescents with $\mathrm{ADHD}$ and might be a complementary treatment option for this disorder.

Keywords: attention deficit/hyperactivity disorder, ADHD, cognitive training, executive functions, intervention, review

Attention Deficit/Hyperactivity Disorder (ADHD) is a neurodevelopmental disorder marked by persistent symptoms of inattention, hyperactivity, and/or impulsivity (American Psychiatric Association, 2013). Children diagnosed with this disorder present difficulties in the ability to pay attention, restrain movements, inhibit impulses, and regulate behavior (Roberts et al., 2015) that affect communication, daily living, and socialization (Weyandt and Gudmundsdottir, 2015). Importantly, according to the literature, these difficulties arise from deficits in executive functioning (e.g., Rapport et al., 2001; Sonuga-Barke, 2003; Willcutt et al., 2005; Nigg, 2006; Barkley, 2015).

Even though there is no agreed-upon definition, executive functions (EF) can be viewed as a multidimensional construct that encapsulates higher-order cognitive processes responsible for guiding, directing, and managing cognitive, emotional, and behavioral functions, particularly during novel problem situations (Gioia et al., 2000). There are several cognitive processes associated with EF, but the major elements include anticipation, goal selection, planning, initiation, self-regulation, mental flexibility, attention, and utilization of feedback (Anderson, 2002). These 
processes develop throughout childhood and adolescence and are invaluable to the cognitive, behavioral, emotional, and social functioning of the individual (Anderson, 2002), originating a variety of difficulties when impaired (Brown, 2013).

Consequently, EF has been broadly investigated in individuals with ADHD and, even though results have been incongruous, studies showed poor performance of children with ADHD on EF tasks when compared to typically developing peers (e.g., Nigg et al., 2002; Willcutt et al., 2005). For instance, a metaanalytic review composed of 83 studies evaluated the validity of the EF theory in this population and observed that children with ADHD display significant deficits in inhibitory control, vigilance, working memory, and planning (Willcutt et al., 2005). A few studies also found difficulties in processing speed (Nigg et al., 2002; Lawrence et al., 2004; Pasini et al., 2007; Yáñez-Téllez et al., 2012), cognitive flexibility (Lawrence et al., 2004; Geurts et al., 2005; Yáñez-Téllez et al., 2012), and sustained attention (Nigg et al., 2002; Trani et al., 2011; Yáñez-Téllez et al., 2012). Thus, problems with EF seem to be hallmark characteristics of this disorder.

Treatment options for ADHD are limited and, frequently, involve the prescription of psychostimulant medication as a first line treatment (Yildiz et al., 2011). Improvements in behavior, attention, interpersonal interactions, cognition (Biederman and Spencer, 2008), and EF (e.g., Barnett et al., 2001; Swanson et al., 2011) reinforce the short-term efficacy of psychostimulant medication, of which methylphenidate and dextroamphetamine are the most prescribed (Rabipour and Raz, 2012). Nevertheless, the limitations of such medication (e.g., short-term effects, unknown long-term effects, sideeffects such as insomnia and lack of appetite) prompt parents and professionals to look for another treatment options (Rabipour and Raz, 2012). Thus, efforts have been made to develop non-pharmacological interventions that decrease ADHD symptomatology, and cognitive training has been considered a potential intervention. As reported by Vinogradov et al. (2012), due to brain plasticity, cognitive training can strengthen and develop essential brain networks and underlying cognitive processes by exposing the brain to well-defined learning tasks, resulting in more adaptive behaviors across contexts.

The tasks presented in cognitive training interventions vary extensively, are usually conferred as games, and can be presented through a computer or pen and paper format, aiming to improve a plethora of abilities such as working memory, attention, inhibitory control, planning, and cognitive flexibility. As it is important to keep the child engaged, motivated, and practicing at a level that is in accordance with or slightly above their current abilities (Diamond, 2012; Rapport et al., 2013), this training is usually adaptive (i.e., the difficulty of the task is adjusted to the performance of the child; Rapport et al., 2013). Thus, it has been proposed that cognitive training can reduce $\mathrm{ADHD}$ symptomatology and improve functioning by addressing the neuropsychological deficits thought to mediate its pathophysiology (Cortese et al., 2015).

A meta-analytic review carried out by Cortese et al. (2015) examined the effects of cognitive training on ADHD symptomatology, neuropsychological deficits, and academic

skills in children and adolescents with ADHD. The authors concluded that there were significant effects of training on ADHD symptoms when considering unblinded raters. Yet, these results were drastically reduced when analyses were limited to trials with active control groups or where raters were blind to treatment conditions. Additionally, significant performance improvements in objective measures of visual and verbal working memory were reported, while there were no effects on inhibition or attention. However, these effects of training on working memory did not extend to academic outcomes. Other reviews found similar results (e.g., Karch et al., 2013; Rapport et al., 2013; Sonuga-Barke et al., 2013). However, the majority of the studies included in these previous reviews focused on a single EF, such as working memory, inhibitory, and attention (or a combination of these). Given that children with ADHD display a diverse set of EF deficits, and training multiple EF might be a more effective strategy than focusing on a single EF domain (Dovis et al., 2015), a systematic literature review focusing on the training of multiple EF domains is lacking.

As it has been proposed that cognitive training for improving executive functioning can reduce ADHD symptomatology (e.g., Cortese et al., 2015), our main aim is to update and extend the findings of previous systematic reviews and meta-analyses, characterizing the current literature on cognitive training interventions for $\mathrm{EF}$ in children and adolescents diagnosed with ADHD. We, thus, examine the outcomes within the included studies to determine the efficacy of cognitive training assessed by performance-based measures of $\mathrm{EF}$ and behavioral/EF questionnaires. Additionally, we intended to evaluate the transfer effects (i.e., generalization of training effects to other nontrained tasks) and possible maintenance of gains reported in each study. As such, we developed the following research question using the PICO framework: In children and adolescents with $\mathrm{ADHD}$, is cognitive training for $\mathrm{EF}$, compared to other types of intervention, typically developing controls, or placebo, effective in developing executive functioning, reducing symptomatology, and improving educational, interpersonal and/or occupational outcomes? (cf. Table 1).

\section{METHODS \\ Search Strategy}

The systematic literature search was conducted between January and February 2018, resorting to EBSCOhost

TABLE 1 | PICO (population/participant, intervention/indicator, comparator/control, outcome) framework.

\section{PICO framework}

Population

Intervention

Comparison

Outcome
Children and adolescents with ADHD, aged 3-14 years old

Cognitive training of at least one domain of executive functioning

Other types of intervention and/or a placebo condition and/or healthy controls

EF, ADHD symptomatology, and/or functional outcomes (i.e., educational, interpersonal, and/or occupational) 


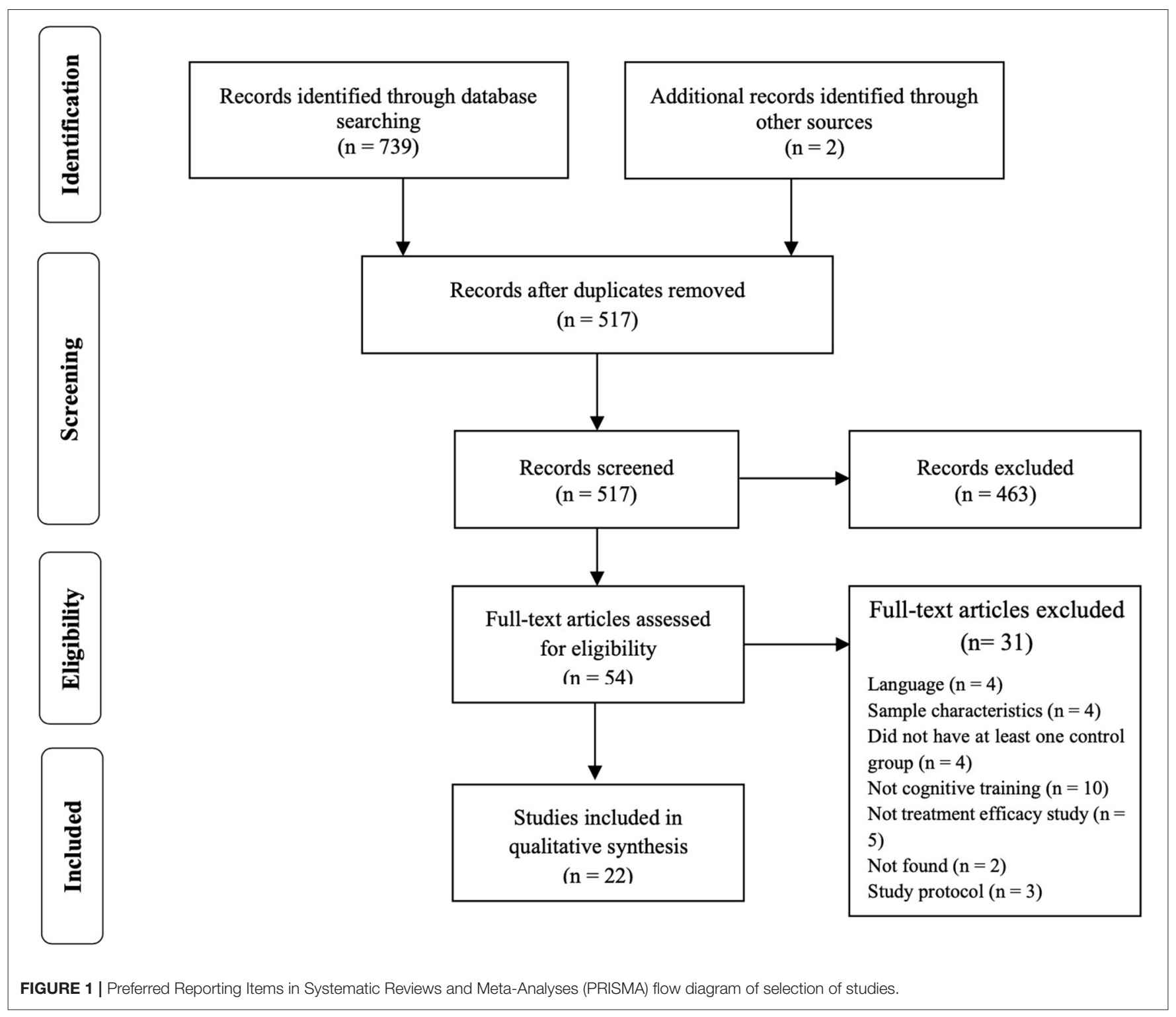

(Academic Search Complete, ERIC, MEDLINE with Full Text, PsycARTICLES, PsycINFO and Psychology and Behavioral Sciences Collection). The search keywords were operationalized using a Population/Participant, Intervention/Indicator, Comparator/Control, Outcome(s) (PICO) search framework (cf. Table 1). Focusing on the last 10 years (2008-2018), the keywords executive function* OR executive functioning AND cognitive training OR intervention* AND Attention Deficit Hyperactivity Disorder OR ADHD were used to conduct the search. Every reference was, then, conveyed to Mendeley and Rayyan, a website developed to assist systematic review authors to perform study selection (Ouzzani et al., 2016), and duplicates were removed. Every study was initially identified by title and abstract, according to the inclusion criteria. Figure 1 details the process of study selection.

\section{Inclusion and Exclusion Criteria}

In order to be included in this review, studies had to: (1) include children with ADHD aged 3-14 years; (2) include a cognitive intervention/training focusing on one or more components of EF; (3) include an active and/or a passive control condition; (4) include measures of EF, ADHD symptomatology, academic achievement and/or interpersonal relationship quality as outcomes; and (5) be published in English.

Reasons for exclusion entailed: (1) reviews, metaanalysis, dissertations, book chapters, and study protocols; (2) studies involving different intervention technics (e.g., mindfulness, neurofeedback); (3) studies focused on different neurodevelopmental disorders (e.g., learning difficulties, Autism Spectrum Disorder) or clinical groups (e.g., Neurofibromatosis); (4) studies including different age ranges (e.g., adults); (5) studies that do not include at least one outcome measure of EF, ADHD 
symptomatology, academic achievement and/or interpersonal relationship quality; and (6), papers not published in English.

Studies were not excluded if children presented comorbid diagnoses or maintained the course of pharmacological treatment during interventions.

After screening each study by title and abstract, the full texts were analyzed and included if they fulfilled the stipulated criteria.

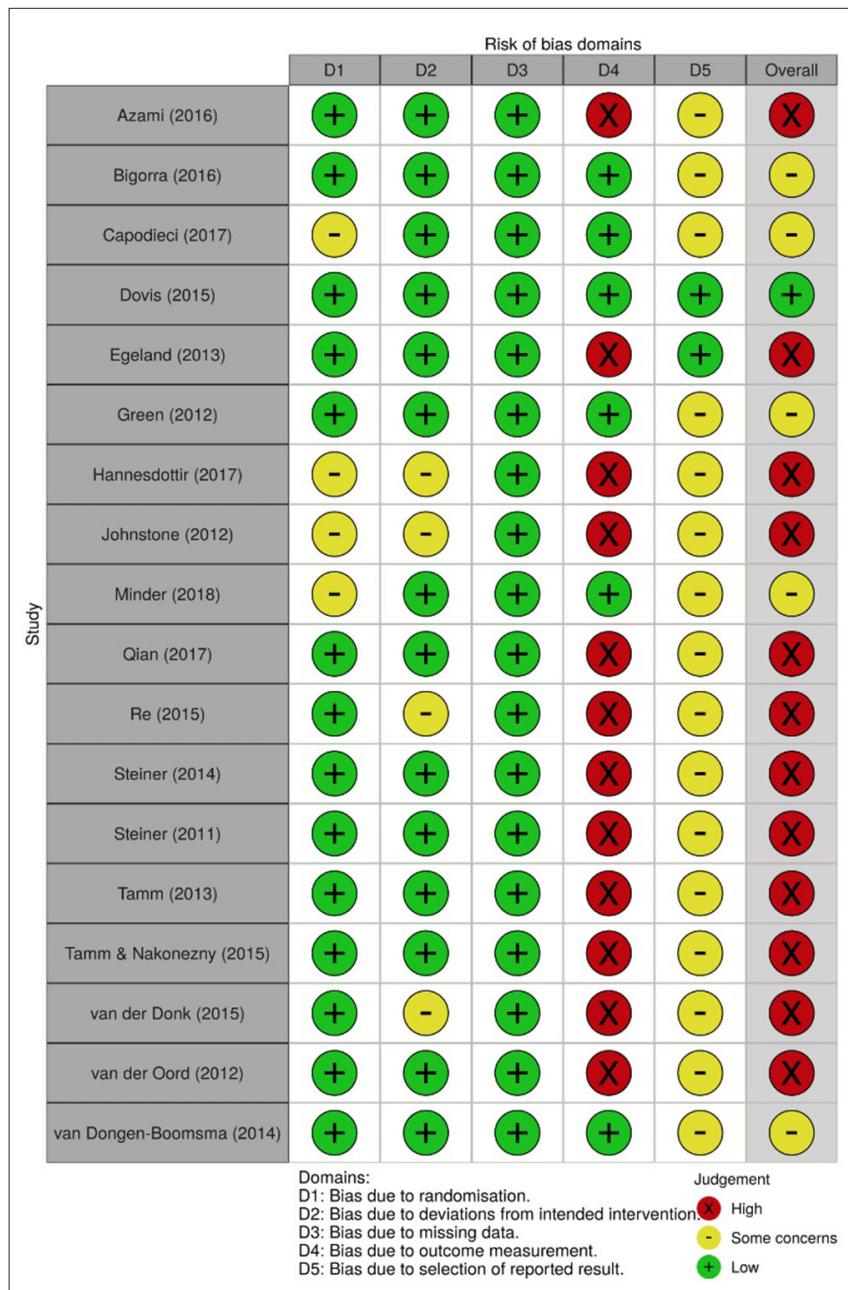

FIGURE 2 | Risk of bias summary for all randomized trials included.
Two additional studies (Green et al., 2012; Johnstone et al., 2012) were hand-searched and included in this review.

\section{Risk of Bias (Quality) Assessment}

In order to assess the risk of bias of the included studies, the Cochrane Collaboration tools were used, namely the RoB 2.0 (Sterne et al., 2019) for the randomized trials and the ROBINS-I (Sterne et al., 2016) for the non-randomized trials. The RoB 2.0 assesses five domains of bias, specifically: (1) bias due to randomization, (2) bias due to deviations from intended intervention, (3) bias due to missing data, (4) bias due to outcome measurement, and (5) bias due to selection of reported result. The ROBINS-I, one the other hand, assesses: (1) bias due to confounding, (2) bias due to selection of participants, (3) bias in classification of interventions, (4) bias due to deviation from intended intervention, (5) bias due to missing data, (6) bias in measurement of outcomes, and (7) bias in selection of the reported result. Risk of bias was independently assessed by AV and MF and disagreements were resolved through discussion. Figures 2-5, designed with the robvis web app (McGuiness, 2019), depict the plots obtained from these analyses.

\section{Data Extraction}

Data obtained from each study encompassed sample characteristics, study design, settings, intervention materials, outcome measures, and main findings.

\section{RESULTS}

\section{Trial Flow}

A total of 739 articles were identified from the databases using the search strategy previously reported. Two additional references were hand-searched and included in this review. Two hundred and twenty-four duplicates were removed, and 517 articles were assessed by title and abstract. Of these, 463 reports were excluded since they did not fulfill the predetermined criteria. Hence, 54 papers were included and their full-text analyzed, of which 22 met inclusion criteria. Again, in Figure 1, the study selection process is presented in a PRISMA flow diagram (Moher et al., 2009).

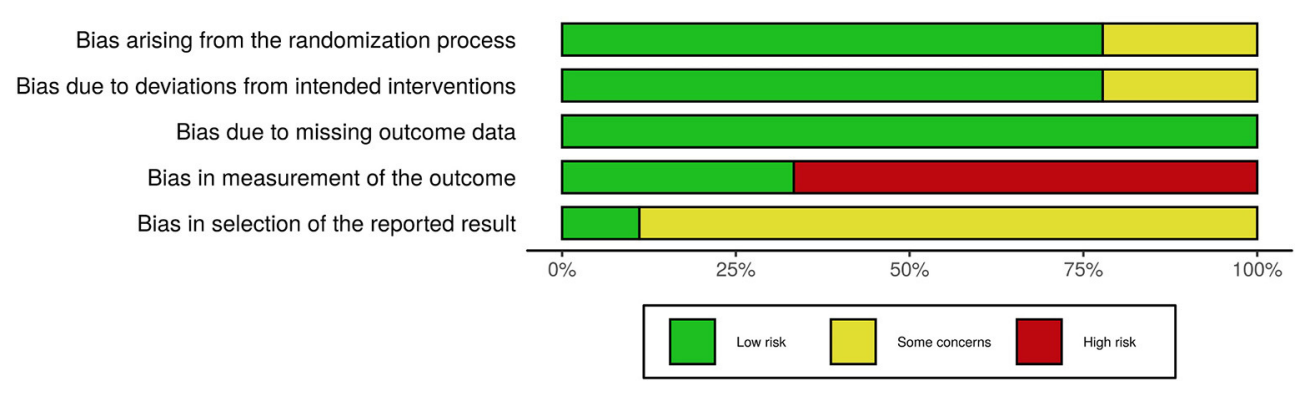

FIGURE 3 | Risk of bias graph for all randomized trials included. 


\section{General Study Characteristics}

The age range of the participants included in each study varied extensively and most of the studies analyzed children aged between 3 and 12 years old (cf. Table 2).

Sample groups were composed by children diagnosed with $\mathrm{ADHD}$ and their typically developing peers. Twelve studies included samples of participants with no comorbidities and seven studies included children with comorbid diagnosis such as Opposition Defiant Disorder (ODD), Conduct Disorder (CD), Learning Disabilities (LD), Anxiety and/or Tics, Mood Disorders, Phobias and/or Elimination Disorders. Three studies did not

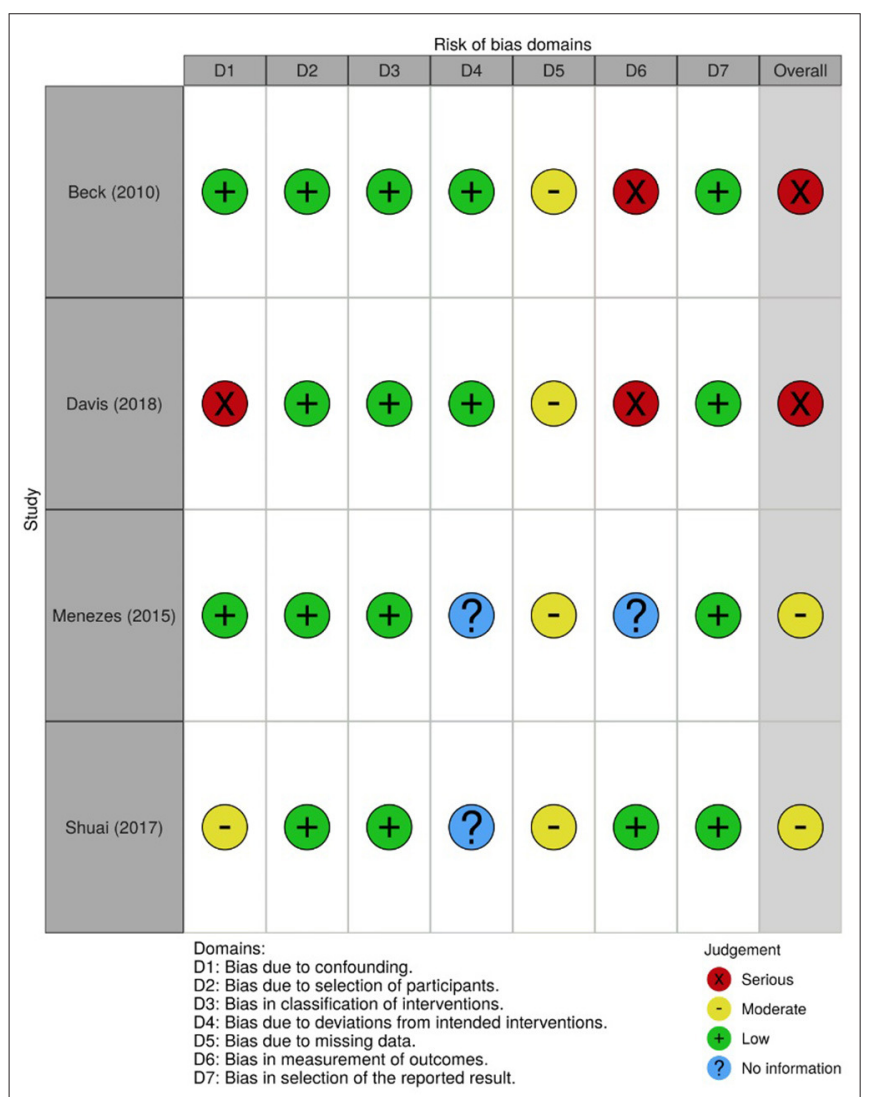

FIGURE 4 | Risk of bias summary for all non-randomized trials included. detail whether they included or excluded participants with comorbidities (cf. Table 2).

Regarding research design, 10 studies were randomized controlled trials, eight were randomized trials, two were open-label/open-trial designs, and two were non-randomized controlled trials (cf. Table 2). In regard to control groups, three of these studies compared the performance of the experimental groups only with active control groups (i.e., medication, nonadaptive training, different intervention) and 14 included passive control groups (i.e., waitlist, healthy control group, normal school activities, treatment as usual) (cf. Table 2). Furthermore, to assess outcomes, eight studies included informants blind to study conditions (i.e., uninformed regarding which people belong to a particular group). One study included only blinded teachers (Beck et al., 2010) and three included blinded classroom observers (Green et al., 2012; Steiner et al., 2014; Minder et al., 2018). In three studies all informants were blind, either parents, teachers, and/or clinicians (van Dongen-Boomsma et al., 2014; Dovis et al., 2015; Bigorra et al., 2016). Lastly, one study used blind clinician assessments (Tamm and Nakonezny, 2015) and another blinded researchers and participants (Green et al., 2012). The remainder fourteen studies used unblinded informants.

Seven studies included in this review were carried out in the USA and 10 in Europe. Two studies were carried out in China, one in Brazil, one in Iran, and one in Australia (cf. Table 2). Eight interventions were conducted at home, six took place in school settings, and four in clinical settings. One study was carried out both in school and clinical settings (Minder et al., 2018). This information was not explicitly stated in three studies. When interventions took place at home, parents where usually the ones providing support during sessions. At school, the intervention was either delivered by teachers, clinicians, or trained research assistants. In regard to the clinical setting, most of the programs were employed by clinicians, but it was not possible to gather precise information in three of these investigations.

Nine of the 22 included studies were published between 2010 and 2014, while five studies were published in 2015, two in 2016, three in 2017, and three in 2018 (cf. Table 2).

\section{Type of Intervention}

The duration of interventions varied extensively. The number of the training sessions varied from 8 to 64 , and each session lasted from $15 \mathrm{~min}$ to $2 \mathrm{~h}$ (average of $52 \mathrm{~min}$ ). The amount of time

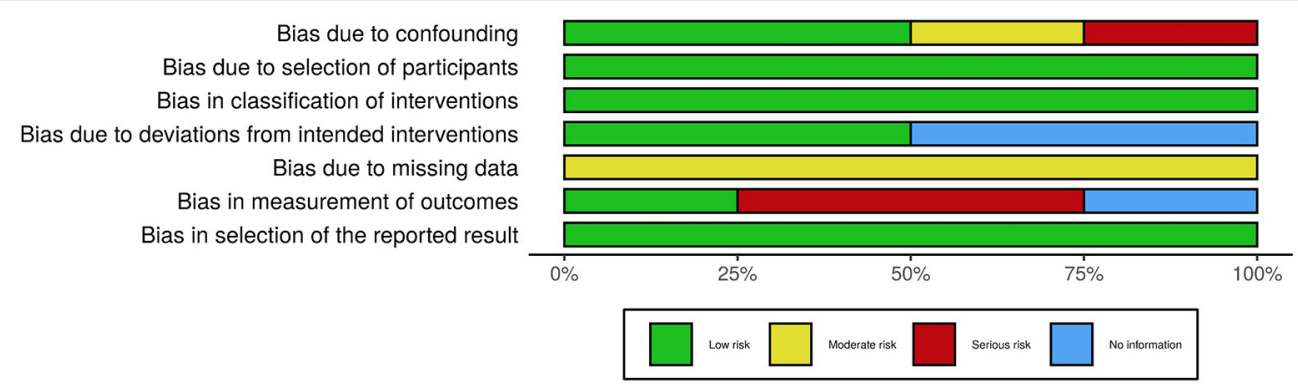

FIGURE 5 | Risk of bias graph for all non-randomized trials included. 
TABLE 2 | Study and intervention characteristics.

\begin{tabular}{|c|c|c|c|c|c|c|c|c|c|c|c|c|c|}
\hline References & Country & Setting & Method & $\begin{array}{l}\text { Age } \\
\text { range }\end{array}$ & Comorbidities & $\begin{array}{l}\text { Type of } \\
\text { comparison } \\
\text { groups }\end{array}$ & $\begin{array}{l}\text { Comparison } \\
\text { groups }\end{array}$ & $\begin{array}{l}\text { Treatment } \\
(n)\end{array}$ & $\begin{array}{l}\text { Comparison } \\
\text { groups }(n)\end{array}$ & Intervention & Duration | Intensity & $\begin{array}{l}\text { Targets of } \\
\text { intervention }\end{array}$ & Outcome measures \\
\hline $\begin{array}{l}\text { Azami et al. } \\
\text { (2016) }\end{array}$ & Iran & Clinical & $\mathrm{RCT}$ & $7-12$ & - & $\begin{array}{l}\text { 1 Active } \\
1 \text { Passive }\end{array}$ & $\begin{array}{l}\text { Medication; } \\
\text { Placebo }\end{array}$ & 12 & $11+11$ & $\begin{array}{l}\text { Persian } \\
\text { software of } \\
\text { working } \\
\text { memory } \\
\text { training; } \\
\text { Lumosity; The } \\
\text { Amazing } \\
\text { Brain Train }\end{array}$ & $\begin{array}{l}20 \times 90 \mathrm{~min} \\
\text { sessions ( } 2 \text { months) }\end{array}$ & $\begin{array}{l}\text { Attention, } \\
\text { inhibition, } \\
\text { short-term } \\
\text { memory, } \\
\text { planning, } \\
\text { processing } \\
\text { speed }\end{array}$ & $\begin{array}{l}\text { CPT, TOL, Digit Span, } \\
\text { RCPM, Span Board, } \\
\text { SNAP-IV }\end{array}$ \\
\hline $\begin{array}{l}\text { Beck et al. } \\
(2010)\end{array}$ & USA & Home & CT & $7-17$ & $\begin{array}{l}\text { ODD; CD; Anxiety; } \\
\text { Mood disorders }\end{array}$ & Passive & Waitlist & 27 & 24 & Cogmed RM & $\begin{array}{l}25 \times 40 \text { min } \\
\text { sessions ( } 6 \text { weeks) }\end{array}$ & $\begin{array}{l}\text { Verbal and } \\
\text { visuo-spatial } \\
\text { working } \\
\text { memory }\end{array}$ & $\begin{array}{l}\text { BRIEF, CRS, number of } \\
\text { DSM-IV-TR inattentive } \\
\text { symptoms }\end{array}$ \\
\hline $\begin{array}{l}\text { Bigorra et al. } \\
\text { (2016) }\end{array}$ & Spain & Home & $\mathrm{RCT}$ & $7-12$ & ODD; CD & Passive & Placebo & 36 & 30 & $\begin{array}{l}\text { Robomemo }{ }^{\circledR} \\
\text { Cogmed } \\
\text { Working } \\
\text { Memory } \\
\text { Training }^{\text {TM }}\end{array}$ & $\begin{array}{l}25 \times 45 \text { min } \\
\text { sessions (5 weeks) }\end{array}$ & $\begin{array}{l}\text { Working } \\
\text { memory }\end{array}$ & $\begin{array}{l}\text { CRS, BRIEF, CBCL, } \\
\text { TRF, SDQ, WFIRF, Digit } \\
\text { Span, Letter-Number } \\
\text { sequencing, Spatial } \\
\text { Span, CPT, TOL, TMT, } \\
\text { WCST }\end{array}$ \\
\hline $\begin{array}{l}\text { Capodieci } \\
\text { et al. (2018) }\end{array}$ & Italy & School & $\mathrm{RT}$ & 5 & - & Passive & Non-training & 38 & 36 & $\begin{array}{l}\text { Activities } \\
\text { presented in } \\
\text { the manual } \\
\text { published by } \\
\text { Caponi et al. }\end{array}$ & $\begin{array}{l}16 \times 60 \text { min } \\
\text { sessions (8 weeks) }\end{array}$ & $\begin{array}{l}\text { Working } \\
\text { memory }\end{array}$ & $\begin{array}{l}\text { IPDDAI; IPDDAG; Span } \\
\text { Backward; Selective } \\
\text { WM; Span Forward; } \\
\text { Walk-No Walk Test; } \\
\text { Matching Figures }\end{array}$ \\
\hline $\begin{array}{l}\text { Davis et al. } \\
\text { (2018) }\end{array}$ & USA & Home & OL/OT & $8-12$ & - & Passive & Non-ADHD & 40 & 40 & Project: EVO & $\begin{array}{l}27 \times 45 \text { min } \\
\text { sessions ( } 6 \text { weeks })\end{array}$ & $\begin{array}{l}\text { Cognitive } \\
\text { interference }\end{array}$ & $\begin{array}{l}\text { Test of Variables of } \\
\text { Attention, BRIEF, } \\
\text { CANTAB }\end{array}$ \\
\hline $\begin{array}{l}\text { Dovis et al. } \\
\text { (2015) }\end{array}$ & Netherlands & Home & $\mathrm{RCT}$ & $8-12$ & - & $\begin{array}{l}1 \text { Active } \\
1 \text { Passive }\end{array}$ & $\begin{array}{l}\text { Partially active } \\
\text { condition; } \\
\text { Placebo }\end{array}$ & 31 & $28+30$ & $\begin{array}{l}\text { Braingame } \\
\text { Brian }\end{array}$ & $\begin{array}{l}25 \times 35-50 \text { min } \\
\text { sessions ( } 5 \text { weeks) }\end{array}$ & $\begin{array}{l}\text { Working } \\
\text { memory, } \\
\text { inhibition and } \\
\text { cognitive } \\
\text { flexibility }\end{array}$ & $\begin{array}{l}\text { Stop task, Stroop test, } \\
\text { CBTT, Digit Span, TMT, } \\
\text { dRCPM, BRIEF, DBDRS }\end{array}$ \\
\hline $\begin{array}{l}\text { Egeland et al. } \\
\text { (2013) }\end{array}$ & Norway & School & $\mathrm{RCT}$ & $10-12$ & - & Passive & $\begin{array}{l}\text { Treatment as } \\
\text { usual }\end{array}$ & 33 & 34 & $\begin{array}{l}\text { Robomemo }{ }^{\circledR} \\
\text { Cogmed } \\
\text { Working } \\
\text { Memory } \\
\text { Training }\end{array}$ & $\begin{array}{l}25 \times 45 \min \\
\text { sessions (5-7 } \\
\text { weeks) }\end{array}$ & $\begin{array}{l}\text { Working } \\
\text { memory }\end{array}$ & $\begin{array}{l}\text { Color Word (CW) and } \\
\text { Trail Making (TMT) tests } \\
\text { from the Delis-Kaplan } \\
\text { Executive Function } \\
\text { System, CPT-II, } \\
\text { Children's Auditory } \\
\text { Verbal Learning Test-2, } \\
\text { Benton Visual } \\
\text { Retention Test-5th } \\
\text { edition, ADHD-Rating } \\
\text { Scale IV, SDQ, BRIEF }\end{array}$ \\
\hline
\end{tabular}


TABLE 2 | Continued

\begin{tabular}{|c|c|c|c|c|c|c|c|c|c|c|c|c|c|}
\hline References & Country & Setting & Method & $\begin{array}{l}\text { Age } \\
\text { range }\end{array}$ & Comorbidities & $\begin{array}{l}\text { Type of } \\
\text { comparison } \\
\text { groups }\end{array}$ & $\begin{array}{l}\text { Comparison } \\
\text { groups }\end{array}$ & $\begin{array}{l}\text { Treatment } \\
(n)\end{array}$ & $\begin{array}{l}\text { Comparison } \\
\text { groups }(n)\end{array}$ & Intervention & Duration | Intensity & $\begin{array}{l}\text { Targets of } 0 \\
\text { intervention }\end{array}$ & Outcome measures \\
\hline $\begin{array}{l}\text { Green et al. } \\
\text { (2012) }\end{array}$ & USA & Home & $\mathrm{RCT}$ & $7-14$ & Unclear & Passive & Placebo & 12 & 14 & WM Cogmed & $\begin{array}{l}25 \times 40 \mathrm{~min} \\
\text { sessions }\end{array}$ & $\begin{array}{l}\text { Working } \\
\text { memory, } \\
\text { off-task } \\
\text { behavior }\end{array}$ & $\begin{array}{l}\text { RAST, CRS, WM index } \\
\text { (Digit-Span and } \\
\text { Letter-Number } \\
\text { Sequence from } \\
\text { WISC-IV) }\end{array}$ \\
\hline $\begin{array}{l}\text { Hannesdottir } \\
\text { et al. (2017) }\end{array}$ & Iceland & - & RT & $8-10$ & Unclear & $\begin{array}{l}\text { 1 Passive } \\
1 \text { Active }\end{array}$ & $\begin{array}{l}\text { Waitlist; Parent } \\
\text { group }\end{array}$ & 16 & $14+11$ & $\begin{array}{l}\text { OutSMARTers } \\
\text { Program }\end{array}$ & $\begin{array}{l}10 \times 2 \mathrm{~h} \text { sessions }(5 \\
\text { weeks) }\end{array}$ & $\begin{array}{ll}\text { Social skills, } & \text { A } \\
\text { self- } & S \\
\text { regulation, } & \text { S } \\
\text { and } & S \\
\text { executive } & \text { V } \\
\text { functions } & (C \\
& \text { a } \\
& \text { S }\end{array}$ & $\begin{array}{l}\text { ADHD Rating Scale-IV, } \\
\text { SSRS, Emotion } \\
\text { Regulation Checklist, } \\
\text { SDQ, Lumosity, } \\
\text { WISC-IV subtests } \\
\text { (Coding, Arithmetic, } \\
\text { and Letter-number } \\
\text { sequence) }\end{array}$ \\
\hline $\begin{array}{l}\text { Johnstone } \\
\text { et al. (2012) }\end{array}$ & Australia & Home & RT & $7-13$ & - & Passive & Waitlist & 83 & 45 & $\begin{array}{l}\text { Feed the } \\
\text { Monkey, Go } \\
\text { Go No-Go }\end{array}$ & $\begin{array}{l}25 \times 20 \text { min } \\
\text { sessions (4-5 } \\
\text { weeks) }\end{array}$ & $\begin{array}{l}\text { Working } \\
\text { memory, } \\
\text { inhibitory } \\
\text { control }\end{array}$ & $\begin{array}{l}\text { Visual Go-No Go task, } \\
\text { Oddball task, Flanker } \\
\text { task, Counting Span, } \\
\text { Digit Span, CRS, } \\
\text { purpose-designed } \\
\text { Behavior Rating Scale }\end{array}$ \\
\hline $\begin{array}{l}\text { Menezes } \\
\text { et al. (2015) }\end{array}$ & Brazil & Clinical & OL/OT & $7-13$ & - & Passive & ADHD & 8 & 10 & PIAFEx & $\begin{array}{l}\approx 64 \times 60 \text { min } \\
\text { sessions ( } 8 \text { months) }\end{array}$ & $\begin{array}{ll}\text { EF and self- } & \mathrm{C} \\
\text { regulation } & \mathrm{T} \\
& \mathrm{A} \\
& \mathrm{A} \\
\mathrm{A} & \mathrm{T}\end{array}$ & $\begin{array}{l}\text { Computerized Stroop } \\
\text { Test, CAT, TMT, WCST, } \\
\text { Auditory WM Test; } \\
\text { Visual WM Test, FAS, } \\
\text { Animals Verbal Fluency } \\
\text { Test, CHEXI }\end{array}$ \\
\hline $\begin{array}{l}\text { Minder et al. } \\
\text { (2018) }\end{array}$ & Switzerland & $\begin{array}{l}\text { Clinical } \\
\text { School }\end{array}$ & RCT & $8-16$ & - & Active & Neurofeedback & 39 & 38 & CogniPlus & (5-14 weeks) & $\begin{array}{l}\text { Attention, } \\
\text { working } \\
\text { memory, } \\
\text { inhibition }\end{array}$ & CRS, BRIEF \\
\hline $\begin{array}{l}\text { Qian et al. } \\
\text { (2017) }\end{array}$ & China & Clinical & RCT & $6-12$ & ODD; LD; Phobias & 2 Passive & $\begin{array}{l}\text { Waitlist; Healthy } \\
\text { control group }\end{array}$ & 38 & $30+23$ & $\begin{array}{l}\text { Based on } \\
\text { Dawson and } \\
\text { Guare's } \\
\text { (2010) training } \\
\text { of executive } \\
\text { skills for } \\
\text { children }\end{array}$ & $\begin{array}{l}12 \times 60 \mathrm{~min} \\
\text { sessions (12 weeks) }\end{array}$ & $\begin{array}{l}\text { Inhibition, S } \\
\text { planning, } \\
\text { working } \\
\text { memory, } \\
\text { time } \\
\text { management, } \\
\text { sustained } \\
\text { attention, } \\
\text { organization, } \\
\text { cognitive } \\
\text { flexibility }\end{array}$ & $\begin{array}{l}\text { Stroop Task, } \\
\text { Rey-Osterrieth } \\
\text { Complex Figure Test, } \\
\text { TMT, BRIEF, } \\
\text { ADHD-RS-IV, WFIRF } \\
\end{array}$ \\
\hline
\end{tabular}


TABLE 2 | Continued

\begin{tabular}{|c|c|c|c|c|c|c|c|c|c|c|c|c|c|}
\hline References & Country & Setting & Method & $\begin{array}{l}\text { Age } \\
\text { range }\end{array}$ & Comorbidities & $\begin{array}{l}\text { Type of } \\
\text { comparison } \\
\text { groups }\end{array}$ & $\begin{array}{l}\text { Comparison } \\
\text { groups }\end{array}$ & $\begin{array}{l}\text { Treatment } \\
(n)\end{array}$ & $\begin{array}{l}\text { Comparison } \\
\text { groups }(n)\end{array}$ & Intervention & Duration | Intensity & $\begin{array}{l}\text { Targets of } \\
\text { intervention }\end{array}$ & Outcome measures \\
\hline $\begin{array}{l}\text { Re et al. } \\
\text { (2015) }\end{array}$ & Italy & School & $\mathrm{RT}$ & 5 & - & Active & Non-training & 26 & $13+13$ & $\begin{array}{l}\text { Development } \\
\text { of } \\
\text { Concentration } \\
\text { and } \\
\text { Self-Control }\end{array}$ & $\begin{array}{l}40 \times 45 \text { min } \\
\text { sessions (5 months) }\end{array}$ & $\begin{array}{l}\text { Attention, } \\
\text { working } \\
\text { memory, and } \\
\text { impulsive } \\
\text { behaviors }\end{array}$ & $\begin{array}{l}\text { Stop-signal test } \\
\text { (Walk-No Walk Test), } \\
\text { The Working Memory } \\
\text { Dual Request Selective } \\
\text { Task, Matching Figures } \\
\text { MF-14, IPPDAl, } \\
\text { IPPDAG }\end{array}$ \\
\hline $\begin{array}{l}\text { Shuai et al. } \\
\text { (2017) }\end{array}$ & China & Clinical & CT & $7-9$ & $\begin{array}{l}\text { ODD; CD; Tics; } \\
\text { Anxiety; Phobias }\end{array}$ & Passive & $\begin{array}{l}\text { Healthy control } \\
\text { group }\end{array}$ & 44 & 88 & $\begin{array}{l}\text { Developed by } \\
\text { the authors }\end{array}$ & $\begin{array}{l}36 \times 45 \mathrm{~min} \\
\text { sessions (4 months) }\end{array}$ & $\begin{array}{l}\text { Inhibition, } \\
\text { working } \\
\text { memory, } \\
\text { planning, } \\
\text { organization, } \\
\text { shifting, } \\
\text { theory of } \\
\text { mind, time } \\
\text { management, } \\
\text { emotional } \\
\text { regulation }\end{array}$ & $\begin{array}{l}\text { Stroop Task, } \\
\text { Rey-Osterrieth } \\
\text { Complex Figure Test, } \\
\text { TMT, BRIEF, ToH, } \\
\text { ADHD Rating Scale-IV, } \\
\text { CRS, false-belief task } \\
\text { t, }\end{array}$ \\
\hline $\begin{array}{l}\text { Steiner et al. } \\
\text { (2014) }\end{array}$ & USA & School & $\mathrm{RCT}$ & $\begin{array}{l}\approx 7-9 \\
\text { 2nd to } \\
4 \text { th } \\
\text { grade }\end{array}$ & - & $\begin{array}{l}1 \text { Passive } \\
1 \text { Active }\end{array}$ & $\begin{array}{l}\text { Treatment as } \\
\text { usual; } \\
\text { neurofeedback }\end{array}$ & 34 & $36+34$ & $\begin{array}{l}\text { BrainTrain } \\
\text { website }\end{array}$ & $\begin{array}{l}40 \times 45 \text { min } \\
\text { sessions (5 months) }\end{array}$ & $\begin{array}{l}\text { Attention, } \\
\text { working } \\
\text { memory }\end{array}$ & $\begin{array}{l}\text { CRS, BRIEF, SKAMP, } \\
\text { Behavioral Observation } \\
\text { of Students in Schools } \\
\text { (BOSS) }\end{array}$ \\
\hline $\begin{array}{l}\text { Steiner et al. } \\
\text { (2011) }\end{array}$ & USA & School & $\mathrm{RT}$ & $\begin{array}{l}\approx 12-14 \\
\text { 6th to } \\
\text { 8th } \\
\text { grade }\end{array}$ & - & $\begin{array}{l}\text { 1 Passive } \\
1 \text { Active }\end{array}$ & $\begin{array}{l}\text { Waitlist; } \\
\text { neurofeedback }\end{array}$ & 13 & $15+13$ & $\begin{array}{l}\text { BrainTrain } \\
\text { website }\end{array}$ & $\begin{array}{l}36 \times 45 \mathrm{~min} \\
\text { sessions (4 months) }\end{array}$ & $\begin{array}{l}\text { Attention, } \\
\text { working } \\
\text { memory }\end{array}$ & $\begin{array}{l}\text { CRS, BRIEF, Behavior } \\
\text { Assessment Scales for } \\
\text { Children-2, Integrated } \\
\text { Visual and Auditory } \\
\text { CPT }\end{array}$ \\
\hline $\begin{array}{l}\text { Tamm et al. } \\
\text { (2013) }\end{array}$ & USA & - & RT & $7-15$ & Unclear & Passive & Waitlist & 54 & 51 & Pay Attention! & $\begin{array}{l}16 \times 30 \text { min } \\
\text { sessions (8 weeks) }\end{array}$ & $\begin{array}{l}\text { Sustained, } \\
\text { selective, } \\
\text { alternating, } \\
\text { and divided } \\
\text { attention }\end{array}$ & $\begin{array}{l}\text { SNAP-IV, BASC-II, CGI, } \\
\text { ATTC, BRIEF, TEA-Ch, } \\
\text { WJ-III, D-KEFS, } \\
\text { Quotient ADHD system }\end{array}$ \\
\hline $\begin{array}{l}\text { Tamm and } \\
\text { Nakonezny } \\
\text { (2015) }\end{array}$ & USA & - & RT & $3-7$ & - & Passive & Waitlist & 13 & 12 & $\begin{array}{l}\text { e.g., } \\
\text { Highlight, } \\
\text { memory card } \\
\text { games }\end{array}$ & $\begin{array}{l}8 \times 60 \text { min sessions } \\
(8 \text { weeks })\end{array}$ & $\begin{array}{l}\text { Attention, } \\
\text { inhibition, } \\
\text { memory, } \\
\text { hand-eye } \\
\text { coordination, } \\
\text { balance, } \\
\text { sensory } \\
\text { awareness, } \\
\text { listening } \\
\text { skills, visual } \\
\text { focusing }\end{array}$ & $\begin{array}{l}\text { BRIEF, CGI, } \\
\text { K-SADS-PL, SNAP-IV, } \\
\text { NEPSY, CELF-IV }\end{array}$ \\
\hline
\end{tabular}




\begin{tabular}{|c|c|c|c|c|c|c|c|c|c|c|c|c|c|}
\hline References & Country & Setting & Method & $\begin{array}{l}\text { Age } \\
\text { range }\end{array}$ & Comorbidities & $\begin{array}{l}\text { Type of } \\
\text { comparison } \\
\text { groups }\end{array}$ & $\begin{array}{l}\text { Comparison } \\
\text { groups }\end{array}$ & $\begin{array}{l}\text { Treatment } \\
(n)\end{array}$ & $\begin{array}{l}\text { Comparison } \\
\text { groups }(n)\end{array}$ & Intervention & Duration | Intensity & $\begin{array}{l}y \text { Targets of } \\
\text { intervention }\end{array}$ & Outcome measures \\
\hline $\begin{array}{l}\text { van der Donk } \\
\text { et al. (2015) }\end{array}$ & Netherlands & School & $\mathrm{RCT}$ & $8-12$ & LD; ODD & Active & $\begin{array}{l}\text { Combined WM- } \\
\text { and EF } \\
\text { compensatory } \\
\text { training }\end{array}$ & 50 & 50 & $\begin{array}{l}\text { Cogmed } \\
\text { Working } \\
\text { Memory } \\
\text { Training, } \\
\text { Paying } \\
\text { attention in } \\
\text { class }\end{array}$ & $\begin{array}{l}25 \times 45 \text { min } \\
\text { sessions ( } 5 \text { weeks) }\end{array}$ & $\begin{array}{l}\text { Working } \\
\text { memory }\end{array}$ & $\begin{array}{l}\text { Creature Counting and } \\
\text { Score!, Digit Span, } \\
\text { Comprehension of } \\
\text { Instruction and Word } \\
\text { List Interference, Span } \\
\text { Board, Six Part test } \\
\text { BADS-C, BRIEF, } \\
\text { CBCL, TRF, }\end{array}$ \\
\hline $\begin{array}{l}\text { van der Oord } \\
\text { et al. (2014) }\end{array}$ & Netherlands & Home & RT & $8-12$ & ODD & Passive & Waitlist & 18 & 22 & $\begin{array}{l}\text { Braingame } \\
\text { Brian }\end{array}$ & $\begin{array}{l}25 \times 50 \text { min } \\
\text { sessions (5 weeks) }\end{array}$ & $\begin{array}{l}\text { Visuospatial } \\
\text { working } \\
\text { memory, } \\
\text { inhibition, } \\
\text { and } \\
\text { cognitive } \\
\text { flexibility }\end{array}$ & BRIEF, DBDRS \\
\hline $\begin{array}{l}\text { van Dongen- } \\
\text { Boomsma } \\
\text { et al. (2014) }\end{array}$ & Netherlands & Home & $\mathrm{RCT}$ & $5-7$ & $\begin{array}{l}\text { ODD; Elimination } \\
\text { disorder; PDDNOS; } \\
\text { DCD; DBNOS }\end{array}$ & Passive & Placebo & 26 & 21 & $\begin{array}{l}\text { Cogmed } \\
\text { Working } \\
\text { Memory } \\
\text { Training }\end{array}$ & $\begin{array}{l}25 \times 15 \text { min } \\
\text { sessions ( } 5 \text { weeks) }\end{array}$ & $\begin{array}{l}\text { Working } \\
\text { memory }\end{array}$ & $\begin{array}{l}\text { ADHD Rating Scale-IV, } \\
\text { BRIEF, Digit Span, } \\
\text { Knox Cubes LDT, } \\
\text { Sentences (WPPSI-R), } \\
\text { RCPM, Day-Night } \\
\text { Stroop task, } \\
\text { SA-DOTS-O2K, Shape } \\
\text { School }\end{array}$ \\
\hline
\end{tabular}

RCT, Randomized controlled trial; RT, Randomized trial; CT, Controlled trial; OP/OT, Open-label/Open-trial; ODD, Opposition Defiant Disorder; CD, Conduct Disorder; LD, Learning Disabilities; PDDNOS, Pervasive Developmental Disorder Not Otherwise Specified; DCD, Developmental Coordination Disorder; DBNOS, Disruptive Behavior Not Otherwise Specified.

CPT, Conners' Continuous Performance Test; TOL, Tower of London; RCPM, Raven's Colored Progressive Matrices; SNAP-IV, Swanson, Nolan and Pelham Questionnaire-4th Edition; BRIEF, Behavior Rating Inventory of Executive Functions; CRS, Conners' Rating Scale; CBCL, Child Behavior Checklist; TRF, Teacher Report Form; SDQ, Strengths and Difficulties Questionnaire; WFIRF, Weiss Functional Impairment Rating Scale; TMT, Trail Making Test; WCST, Wisconsin Card Sorting Test; IPDDAl, Early Identification of ADHD for Parents; IPDDAG, Early Identification of ADHD for Teachers; CANTAB, Cambridge Neuropsychological Test Automated Battery; CBTT, Corsi Block Tapping Test; DBDRS, Disruptive Behavior Disorders Rating Scale; RAST, Restricted Academic Setting Task; SSRS, Social Skills Rating System; WISC, Wechsler Intelligence Scale for Children; FAS, Phonological Fluency Test; CHEXI, Childhood Executive Functioning Inventory; ToH, Tower of Hanoi; SKAMP, Swanson, Kotkin, Agler, M-Flynn and Pelham Scale; CGI, Clinical Global Impressions; WJ-III, Woodcock-Johnson Tests of Achievement-3rd edition; D-KEFS, Delis-Kaplan Executive Functioning System; BASC-II, Behavioral Assessment System for Children-2nd edition; ATTC, Attentional Control Scale; TEA-ch, Test of Everyday Attention for Children; K-SADS-PL, Kiddie Schedule for Affective Disorders and Schizophrenia for school-aged children - Present and Lifetime Version; NEPSY, Developmental Neuropsychological Assessment Battery; CELF-IV, Clinical Evaluation of Language Fundamentals-4th edition; WPPSI-R, Wechsler Preschool and Primary Scale of Intelligence; SA-DOTS-02K, Sustained Attention Dots Task. 
participants spent in training ranged between 375 and 3,840 min (average of 1,096 min per intervention).

Two types of intervention materials were considered: computerized (Beck et al., 2010; Steiner et al., 2011, 2014; Green et al., 2012; Johnstone et al., 2012; Egeland et al., 2013; van der Oord et al., 2014; van Dongen-Boomsma et al., 2014; Dovis et al., 2015; Azami et al., 2016; Bigorra et al., 2016; Davis et al., 2018; Minder et al., 2018) and non-computerized cognitive training (Tamm et al., 2013; Menezes et al., 2015; Re et al., 2015; Tamm and Nakonezny, 2015; Hannesdottir et al., 2017; Qian et al., 2017; Shuai et al., 2017; Capodieci et al., 2018). One study used both types of intervention in order to compare their efficacy (van der Donk et al., 2015).

Regarding the computerized training, the majority of the studies employed some variation of the Cogmed software. Additional websites and software encompass the Persian software of working memory training, Project: Evo, Braingame Brian, CogniPlus, the Brain Train website, and others. Further programs and activities, without computerized characteristics, feature the activities presented in the manual published by Caponi and collaborators, the OutSMARTers program, PIAFEx, a training program based on Dawson and Guare's training of executive skills for children, and other activities. One study did not specify the training program used during the intervention but provided some insights about its characteristics (Shuai et al., 2017).

\section{Targets of Intervention}

As previously stated, there is no universally accepted definition of $\mathrm{EF}$ and, therefore, there is no agreement concerning the elements incorporated in this construct. Consequently, there is extensive variability in the domains of intervention across studies, and, in total, $16 \mathrm{EF}$ were considered. Five of these 16 domains were targeted more frequently, specifically: attention $(n=8)$, inhibition $(n=8)$, working memory $(n=16)$, planning $(n=3)$, and cognitive flexibility $(n=4)$. Other components of executive functioning addressed by interventions incorporate organization, processing speed, short-term memory, self/emotional regulation, time management, and theory of mind (cf. Table 2).

\section{Outcome Measures}

A variety of measures were used across studies to measure EF, as detailed in Table 3. These measures can be subdivided into two categories: (1) performance-based neuropsychological measures (i.e., computer-oriented or pen-paper tasks) and (2) behavioral and EF questionnaires (under the perspective of parents, teachers, clinicians and/or significant others).

Within the 22 studies, 44 performance-based measures were used to assess particular aspects of EF. Of these 44 measures, 10 were adopted in three or more studies, respectively: (1) Conners' Continuous Performance Test, (2) Trail Making Test, (3) Wisconsin Card Sorting Task, (4) Delis-Kaplan Executive Functioning System, (5) Stroop Test, (6) Rey-Osterrieth Complex Figure, (7) Digit Span, (8) Raven Colored Progressive Matrices, (9) Developmental Neuropsychological Assessment Battery, and (10) Letter-Number Sequencing. It is important to note, however, that not all studies included in this review used performancebased measures to verify the efficacy of their intervention.

TABLE 3 | Performance-based measures and questionnaires employed across studies.

Performance-based measures of attention and EF

Conners Continuous Knox Cubes LDT

Performance Test

Tower of London

Digit Span

Raven Colored Progressive

Matrices

Span Board

Trail Making Test

Auditory Working Memory Test

Visual Working Memory Test

Wisconsin Card Sorting Task

Spatial Span

Cambridge Neuropsychological

Test Automated Battery

Test of Variables of Attention

Stop Task

Tower of Hanoi

Woodcock-Johnson Tests of

Achievement-3rd edition

Stroop Test

Corsi Block Tapping Test

Delis-Kaplan Executive

Functioning System

Rey-Osterrieth Complex Figure

Test of Everyday Attention for

Children

Quotient ADHD System

Shape School

Cancellation Attention Test

Phonological Fluency Test

Animals Verbal Fluency Test

Developmental Neuropsychological Battery

Clinical Evaluation of Language

Fundamentals-4th edition

Walk/No-Walk Test

Selective Working Memory Test

Matching Figures Test (MF-14)

Visual Go/No-Go Task

Oddball Task

Flanker Task

Counting Span

The Working Memory Dual Request Selective Task

Letter-number Sequencing (WISC-III/IV)

Coding (WISC-IV)

Arithmetic (WISC-IV)

Creature Counting and Score!

Comprehension of Instruction and Word List Interference

Sentences (WPPSI-RN)

Sustained Attention Dots Task

Behavioral Assessment of the Dysexecutive Syndrome in Children

Ratings of symptomatology and EF
Swanson, Nolan and Pelham Questionnaire-4th Edition

Behavior Rating Inventory of Executive Function

Conners Rating Scales

Child Behavior Checklist

Teacher Report Form

Strengths and Difficulties

Questionnaire

WEISS Functional Impairment

Rating Scale

Disruptive Behavior Disorders

Rating Scale

ADHD Rating Scale IV

Emotion Regulation Checklist
Behavior Assessment Scales for Children-2

Clinical Global Impressions

Childhood Executive Functioning Inventory

Attentional Control Scale

Swanson, Kotkin, Agler, M-Flynn and Pelham Scale

IPDDAI (Early Identification of ADHD for Parents)

IPDDAG (Early Identification of ADHD for Teachers)

Kiddie Schedule for Affective Disorders and Schizophrenia for school-aged children Social Skills Rating System
Kidscreen-27

Furthermore, within the 20 ratings employed, five were widely used across studies, specifically: (1) Behavior Rating Inventory of Executive Functions, (2) Conners' Rating Scales, (3) Swanson, Nolan, and Pelham Questionnaire-4th edition, (4) Strengths and Difficulties Questionnaire, and (5) ADHD Rating Scale-IV. 


\section{Effects of Intervention}

Data for EF outcomes were examined in each study in order to determine the efficacy of the interventions, and details are outlined in Table 4. Of the 22 studies included in this review, 14 reported improvements in performance-based measures of EF (Green et al., 2012; Johnstone et al., 2012; Egeland et al., 2013; Tamm et al., 2013; Dovis et al., 2015; Menezes et al., 2015; Re et al., 2015; van der Donk et al., 2015; Azami et al., 2016; Bigorra et al., 2016; Shuai et al., 2017; Capodieci et al., 2018; Davis et al., 2018), four didn't resort to these measures to evaluate the outcomes (Beck et al., 2010; Steiner et al., 2014; van der Oord et al., 2014; Minder et al., 2018), four were unable to find significant differences between groups (van Dongen-Boomsma et al., 2014; Tamm and Nakonezny, 2015; Hannesdottir et al., 2017; Qian et al., 2017), and one was unclear regarding its findings (Steiner et al., 2011). In the 14 studies that presented positive results in performance-based measures, improvements were reported for attention (Johnstone et al., 2012; Tamm et al., 2013; van der Donk et al., 2015; Azami et al., 2016; Bigorra et al., 2016; Davis et al., 2018), working memory (Green et al., 2012; Dovis et al., 2015; Menezes et al., 2015; Re et al., 2015; van der Donk et al., 2015; Shuai et al., 2017; Capodieci et al., 2018; Davis et al., 2018), inhibition (Dovis et al., 2015; Menezes et al., 2015; van der Donk et al., 2015; Azami et al., 2016; Bigorra et al., 2016; Davis et al., 2018), visuospatial short-term memory (Dovis et al., 2015; Azami et al., 2016), verbal shortterm memory (Azami et al., 2016), attentional control (Re et al., 2015; Capodieci et al., 2018), interference control (Dovis et al., 2015; Shuai et al., 2017), impulsiveness (Re et al., 2015; Capodieci et al., 2018), processing speed (Egeland et al., 2013; Shuai et al., 2017), shifting (Shuai et al., 2017), planning (Tamm et al., 2013; Shuai et al., 2017), and reasoning (Azami et al., 2016). The effect sizes reported ranged from small to large (cf. Table 4 for detailed results).

Regarding the ability of cognitive training to trigger change in day-to-day life (i.e., transfer effects; Toplak et al., 2008), 11 studies have shown decreases in parent and/or teacher ratings of ADHD symptomatology and 11 studies conveyed reductions on EF difficulties according to informants, with small to large effect sizes (cf. Table 4). Studies also stated improvements in social skills (Hannesdottir et al., 2017; Qian et al., 2017), progress in academic performance (Egeland et al., 2013; Qian et al., 2017; Shuai et al., 2017), and reduced off-task behavior (Green et al., 2012; Minder et al., 2018; cf. Table 4). In the matter of the assessment of EF behaviors in everyday environments, informants reported positive changes in working memory (Beck et al., 2010; Tamm et al., 2013; Tamm and Nakonezny, 2015; Bigorra et al., 2016; Shuai et al., 2017), initiation (Beck et al., 2010; Tamm et al., 2013; Shuai et al., 2017), planning/organization (Beck et al., 2010; Tamm et al., 2013; Tamm and Nakonezny, 2015; Shuai et al., 2017), monitoring (Tamm et al., 2013; Bigorra et al., 2016; Shuai et al., 2017), shifting (Tamm et al., 2013; Bigorra et al., 2016), inhibition (Tamm and Nakonezny, 2015; Shuai et al., 2017), emotional control (Shuai et al., 2017), the metacognition (Tamm et al., 2013; van der Oord et al., 2014; van der Donk et al., 2015; Bigorra et al., 2016; Shuai et al., 2017; Minder et al., 2018) and behavioral regulation indexes (Tamm et al., 2013; van der Donk et al., 2015; Shuai et al., 2017; Minder et al., 2018), and the global executive composite (Steiner et al., 2011; Tamm et al., 2013; van der Oord et al., 2014; Shuai et al., 2017).

Of these 22 studies, $13 \mathrm{did}$ not analyze the possible maintenance of gains through follow-up sessions. Of the nine studies that performed follow-up sessions, seven concluded that the gains observed at the end of the intervention were maintained throughout time (Beck et al., 2010; Johnstone et al., 2012; Egeland et al., 2013; van der Oord et al., 2014; van der Donk et al., 2015; Bigorra et al., 2016; Hannesdottir et al., 2017).

In order to assess if study design had implications in the results obtained across studies, a qualitative comparison of the results obtained in randomized $(n=18)$ vs. non-randomized $(n=4)$ trials was conducted. On one hand, randomized studies reported, more often than non-randomized trials, improvements in ratings ( $\simeq 33$ vs. $\simeq 25 \%$ ) as well as in performance-based measures and ratings combined ( $\simeq 33$ vs. $\simeq 25 \%$ ). On the other hand, nonrandomized trials reported improvements only in performancebased measures more frequently than randomized studies $(\simeq 50$ vs. $\simeq 22 \%$ ).

As previously reported, seven studies contemplated in this review included samples of children with comorbid diagnoses. A qualitative comparison of the results obtained by these studies showed that, comparatively to studies that included children with an ADHD diagnosis only, a higher proportion of studies with included comorbid diagnoses reached improvements in ratings $(\simeq 43$ vs. $\simeq 25 \%$ ) and performance-based measures in combination with ratings $(\simeq 43$ vs. $\simeq 25 \%$ ). Also, in samples without comorbid diagnoses, improvements were found more frequently only in performance-based measures $(\simeq 42 \%)$.

\section{DISCUSSION}

The first aim of this study was to update and extend the findings of previous reviews, characterizing the current literature on cognitive training interventions for $\mathrm{EF}$ in children and adolescents diagnosed with ADHD between 3 and 14 years of age. A total of 741 articles were identified and, after duplicates removal, 517 articles were analyzed. Twenty-two studies were eligible for inclusion. Regarding the characteristics of the included studies, it is useful to highlight important methodological features. Studies tend to include individuals diagnosed with ADHD and other comorbid disorders (i.e., ODD, CD, LD, Anxiety and/or Tics, Mood Disorders, Phobias, and/or Elimination Disorders). Regarding research designs, the majority of the included studies were randomized trials, but a few non-randomized studies were also included as they fulfilled the pre-established inclusion criteria. Also, although all studies included control groups, unblinded outcomes assessments were performed frequently. Most of the research was conducted in USA and Europe in home, school, and/or clinical settings. Regarding intervention programs, computerized but also noncomputerized programs were frequently employed. The most frequent EF domains targeted by interventions were attention, inhibition, working memory, planning, and cognitive flexibility.

Our second aim was to assess whether cognitive training was effective in ADHD as evaluated by performance-based measures of $\mathrm{EF}$ and/or behavioral/EF questionnaires. Results showed that most of the studies that used performance-based measures 
TABLE 4 | Results of included studies.

\begin{tabular}{|c|c|c|c|}
\hline & Study & Effe & sizes \\
\hline 1 & Azami et al. (2016) & $\begin{array}{l}\text { CPT (total correct): } d=1.12 \\
\text { Raven's progressive matrices: } d=1.436 \\
\text { Backward digit span: } r= \\
-0.721 \text { (placebo); } r=-0.11 \text { (medication) } \\
\text { SNAP-IV (ADHD-PHI): } d=1.784\end{array}$ & $\begin{array}{l}\text { Span board: } d=1.34 \\
\text { Forward digit span: } r=-0.567 \text { (placebo); } \\
r=-0.037 \text { (medication) } \\
\text { SNAP-IV (ADHD-C): } d=1.422\end{array}$ \\
\hline 2 & Beck et al. (2010) & $\begin{array}{l}\text { ADHD index: } d=0.76 \\
\text { Cognitive problems/inattention: } d=0.79 \\
\text { Hyperactivity: } d=0.36 \\
\text { DSM-IV inattentive scale: } d=1.49 \\
\text { BRIEF Teacher Scale }\end{array}$ & $\begin{array}{l}\text { BRIEF Parent Scale } \\
\text { Metacognition index: } d=0.91 \\
\text { Working memory: } d=0.85 \\
\text { Initiate: } d=0.94 \\
\text { Plan|organize: } d=0.92\end{array}$ \\
\hline
\end{tabular}

$3 \quad$ Bigorra et al. (2016)

Working memory composite score: $d=$ 0.81

CPT (detectability): $d=0.60$

BRIEF Parent Scale

Working memory: $d=-0.86$

Plan|organize: $d=-0.71$

Metacognition index: $d=-0.78$

ADHD symptom composite

Parents: $d=-0.39$

Teachers: $d=-0.69$

Forward digit span: $d=0.72$

Backward digit span: $d=1.70$

Selective working memory: $d=1.70$

CPT (commission errors): $d=0.40$ BRIEF

Teacher Scale

Initiate: $d=-0.55$

Working memory: $d=-0.36$

Monitor: $d=-0.72$

Shift: $d=-0.39$

Metacognition: $d=-0.37$

School learning behavior (WFIRS-P): $d$

$=-0.86$

Walk-No walk: $d=1.25$

MF-14: $d=1.29$

\section{Test of Variables of Attention} Attention Performance Index: $d=0.35$ Attention Performance Index (high severity): $d=0.71$

\section{Main findings}

For simple EF tasks (e.g., sustained attention and inhibition), the experimental group had the same results as the active stimulant medication group. However, for a number of complex EFs (e.g., verbal and visuospatial short-term memory and non-verbal reasoning), the experimental group showed better results than the active stimulant medication and placebo groups.

The experimental group showed better results on parent ratings of overall ADHD symptoms, inattention, initiation, planning/organization, and working memory than the waitlist control group.

The experimental group improved significantly more than the control group on parent ratings of the metacognition index (i.e., the child's ability to monitor, initiate, plan, organize, and sustain future-oriented problem solving in working memory). Also, the experimental group improved significantly more than the control group on teacher ratings of the metacognitive index, initiation, working memory, monitoring, and shifting. Also, for the experimental group compared to the control group there were significant improvements in performance-based measures of EF, ADHD symptoms, and functional impairment.

The experimental group showed better results than the control group in performance-based measures of working memory and other neuropsychological measures. Effects were not found for inattention and hyperactivity problems rated by teachers and parents.

The experimental group showed more improvements than the control group on performance-based measures of attention, working memory, and inhibition than the control group, especially among children with greater symptom severity and impaired attention.

Only children in the full-active condition (where working memory, inhibition, and cognitive flexibility were trained) compared to a partially-active condition (where only inhibition and cognitive flexibility were trained) and to a placebo condition showed better results on measures of visuospatial short-term memory and working memory.

The experimental group presented better results than the control group only in processing speed. Reading and mathematics were improved in the experimental group, changes in ADHD symptom rating scales were not visible. In addition, the improvements in reading scores remained significant 8 months later.

The experimental group presented reductions in off-task ADHD-associated behaviors after training. Improvements in working memory performance-based measures were also found. No significant improvements were found on parent rating scales. 
TABLE 4 | Continued

Study (2017)

10

Johnstone et al. (2012)
Effect sizes

Social Skills Rating System

Total score: $d=0.54$ Emotion

Inattention: $d=0.90$

Hyperactivity|Impulsivity: $d=0.74$

Strengths and Difficulties Questionnaire

Total score: $d=0.75$

Emotion regulation: $d=0.67$

Conners-3 ADHD DSM-IV indices (Parent) Inattention: $\eta^{2}=0.096$

MF-14 (errors): $\eta_{p}^{2}=0.37$

Main findings

Compared to a waitlist control group, the experimental group (OutSMARTers

Program) showed a reduction of ADHD symptomatology, improved social skills and better emotion regulation according to parents. No improvements were

found on performance-based measures. No differences were found between the experimental and a parent training group, as both groups showed some improvement. These improvements were still visible 3 months later.

Children in both experimental groups (i.e., working memory and inhibitory control training with and without attention monitoring) showed significant improvements in ratings of $A D H D$ symptomatology according to parents and other family members. Better performance in tasks pertaining to spatial working memory, ignoring distracting stimuli, and sustained attention were also reported, with the attention monitoring via EEG retaining little effect on the outcomes. The follow-up sessions carried after a 6-week interval revealed maintenance of gains.

The experimental group showed better performance on measures of attention/inhibition and auditory working memory compared to the control group. No effect was found for measures of more complex executive functions, such as flexibility, visual working memory, and verbal fluency. Parent rating scales showed no improvement of ADHD symptomatology or executive functioning.

Both experimental groups (cognitive training vs. neurofeedback) improved in ratings of $A D H D$ symptomatology and executive functions according to parents and teachers and off-task behavior as reported by blinded raters. An effect of training was found for cognitive training only on inattention symptoms rated by parents.

After the intervention, children in the experimental group were rated by parents as displaying improved executive functioning, diminished ADHD symptomatology, reduced risk-tasking behaviors and enhanced academic performance. Despite these improvements, the experimental group was still distinguishable from the healthy control group in almost all variables.

Children with ADHD presented better performance in tasks assessing attention, inhibition, and working memory. Improvements in children with typical development who attended the training were also found. Both parents and teachers' ratings of ADHD symptomatology improved for the experimental and control groups.

The experimental group presented better performance in neuropsychological tests after the intervention, with improvements in processing speed, inhibition, shifting, working memory, and planning. Results from parent rating scales showed reduced ADHD symptomatology and behavioral problems as well as improved executive functioning and academic performance. At post-test, there were no significant differences between the ADHD and healthy control groups.

Only children who attended the neurofeedback intervention showed significant improvement in ratings of attention, executive functioning and off-task behavior compared with those in the control and cognitive training conditions. 
TABLE 4 | Continued

\begin{tabular}{lll}
\hline \multicolumn{1}{l}{ Study } & \\
\hline $17 \quad$ Steiner et al. (2011) & Conners Rating Scales-Revised \\
& Inattention: $d=0.80$ \\
& ADHD index: $d=-0.70$ \\
& \\
& \\
& \\
& \\
& & \\
& SNAP-IV (Parent) \\
& Inattention: $d=1.65$ \\
& Hyperactivity||mpulsivity: $d=0.65$ \\
& SNAP-IV (Clinician) \\
& Inattention: $d=1.41$ \\
& Hyperactivity||mpulsivity: $d=0.68$ \\
& Behavior Assessment Scales for \\
& Children-2 \\
& Attention Problems (parent): $d=0.66$ \\
& Clinical Global Impressions \\
& Severity: $d=1.04$ \\
& Improvement: $d=1.14$ \\
& BRIEF Parent Scale \\
& Shift: $d=1.01$ \\
& Emotion Regulation: $d=0.97$
\end{tabular}

20 van der Donk et al. (2015)

van der Oord et al. (2014)
Effect sizes

Behavior Assessment Scales for Children $-2$

Attention Problems: $d=-0.80$

Behavior Rating Inventory of

Executive Function

Global Executive Composite: $d=$

$-0.60$

D-KEFS Tower time per move ratio: $d$

$=0.55$

BRIEF Parent Scale

Shift: $d=0.63$

Initiate: $d=0.98$

Working memory: $d=1.16$

Planning: $d=1.00$

Organization: $d=0.53$

Monitor: $d=0.70$

Behavioral Regulation Index: $d=0.63$

Metacognition Index: $d=1.13$

General Executive Composite: $d=1.03$

SNAP-IV (Clinician)

Inattention: $d=1.10$

Span board: $d=0.85$

Creature counting (correct): $d=0.26$

Word list interference (remember): $d=$

$-0.33$

BRIEF Teacher Scale

Metacognition Index: $d=-0.07$

Disruptive Behavior Disorder Rating Scale Inattention: $\eta^{2}=0.25$

Hyperactivity||mpulsivity: $\eta^{2}=0.22$
Main findings

The experimental group that received Neurofeedback training was rated by parents as presenting less symptoms of ADHD and improved behavior. Parents of children that attended the Standard Computer Format training reported less inattention and ADHD symptoms as well as improvements in executive functioning. Teacher and self-report ratings did not show symptomatology improvements.

After the intervention, the experimental group performed significantly better on a measure of planning compared to a waitlist control group. No effects were found in the remainder performance-based measures. Parents rated children in the experimental group as presenting fewer ADHD symptoms and better executive functioning. Clinician ratings of ADHD symptoms presented reduced scores and children reported a better ability to focus and shift attention. Teacher ratings did not reach statistical significance.

No improvements were found in performance-based measures of executive functions for the experimental group compared to the waitlist control group following the intervention. However, parents of children in the experimental group reported effects on the shift and emotion regulation subscales of the BRIEF. Blinded clinicians' ratings revealed decreased inattention symptoms.

Both experimental groups (Cogmed working memory training vs. Pay Attention in Class) improved on measures of attention, inhibition, and planning. Parent and teacher ratings of executing functioning and $\mathrm{ADHD}$ symptomatology presented decreased scores, but no effects were found on academic, behavioral, and quality of life outcomes.

The experimental group showed better results on parent ratings of ADHD symptomatology as well as on the metacognition index and total score of the BRIEF compared to a waitlist control group. These effects maintained stable at the 9-week follow-up and improvements on inhibition were found. Teacher ratings showed no effects of training at post-test but revealed improvements from pre-test to follow-up on ADHD symptomatology.

No significant treatment effect was found on the outcome measures applied. The experimental and placebo groups did not differ at the end of the intervention on behavioral symptoms, neurocognitive performance, executive and global functioning. 
demonstrated efficacy in improving one or more domains of EF in children and adolescents. Study design appears to have implications in the results obtained across studies, as a qualitative comparison of the results obtained in randomized and nonrandomized trials showed that randomized studies reported, more often than non-randomized trials, improvements in ratings as well as in performance-based measures and ratings combined. Conversely, non-randomized trials reported improvements only in performance-based measures more frequently than randomized studies.

Furthermore, we intended to evaluate the transfer of gains (i.e., the generalization of training effects to other nontrained tasks). Regarding these transfer effects, research has shown decreases in parent and/or teacher ratings of ADHD symptomatology, social skills improvements, and reductions in $\mathrm{EF}$ dysfunction in daily life. Additionally, improvements in academic performance and reduced off-task behavior (i.e., task disengagement to engage in unrelated behaviors) were reported. In spite of these results showing that cognitive training can be an effective intervention for children and adolescents with ADHD, our conclusions should be interpreted considering limitations of the included studies as discussed below.

As previously mentioned, several studies integrated in this review included participants with associated comorbidities. In fact, Efron et al. (2016) found that in a sample of 132 diagnosed children, aged $4-7$ years, $39 \%$ had one comorbidity and $37 \%$ had more than one comorbidity. According to the authors, ODD (53\%), Anxiety Disorder (23.5\%), LD (15.9\%), and Language Disorder (14.4\%) were the most common comorbidities across individuals with ADHD. Similar results have been reported by Reale et al. (2017). The authors found that of the 1,919 subjects evaluated, $66 \%$ had at least one comorbid diagnosis, while only $34 \%$ presented just ADHD. Among the most common comorbid diagnosis were LD (56\%), Sleep Disorders (23\%), ODD (20\%), and Anxiety Disorders (12\%). Therefore, it is clear that the majority of children diagnosed with $\mathrm{ADHD}$ have, at least, one comorbid disorder.

The comparison of the results obtained by the included studies in this review demonstrated that a higher proportion of studies that included comorbid diagnoses reached improvements in ratings and performance-based measures in combination with ratings. Contrarily, in samples without comorbid diagnoses, improvements were found more frequently only in performancebased measures. These results suggest that, for children with comorbidities, improvements perceived by informants in daily life were more frequent. In fact, as discussed by Diamond (2012), children with greater difficulties on executive functioning may benefit the most from any intervention focusing on its training. As children with comorbid diagnoses usually present with higher levels of symptomatology and EF problems, they may have more room for improvement than children presenting with one single diagnosis (Flook et al., 2010). As such, individuals with different comorbidities may respond differently to specific treatments, have differing clinical correlates, and/or demonstrate unique clinical outcomes what might influence results obtained across studies. So, additional studies should group ADHD individuals into more homogenous subgroups based on comorbid patterns.
Regarding control groups, in order to consider that differences between the groups may be accounted to effects of the intervention, researchers should compare the performance of an experimental group and a control group that "accounts for improvements caused by factors other than the treatment" (i.e., an active control group; Boot et al., 2013). In line with these theoretical assumptions, assessing the efficacy of interventions by comparing the performance between a treated group and a non-treated group (i.e., treatment as usual, waitlist, typical development) would not be appropriate since both groups have different expectations (Boot et al., 2013), influencing results on outcome measurements. In fact, of the studies included in this review, three reported active control groups and five used both active and passive control groups. Nevertheless, the majority of the comparison groups were passive $(n=14)$, and these considerations should be taken into account while interpreting these results.

Another limitation of most of the studies conducted is the lack of transfer effects when the efficacy of the intervention is assessed by blinded raters. Even though this was confirmed across the majority of studies that included unblinded informants, positive results should also be highlighted. Beck et al. (2010) and Bigorra et al. (2016) assessed treatment efficacy through blinded raters and found significant results. Beck et al. (2010) found slight improvements in the initiate scale of the BRIEF-Teacher Form, even though these only approached significance. Bigorra et al. (2016) found significant improvements in several subscales of the BRIEF-Teacher and Parent Forms, with small to large effect sizes. The authors also found significant improvements in ADHD symptoms according to both teachers and parents. These results show that blind raters are able to detect changes in everyday situations following cognitive training interventions. Nevertheless, more studies using blind raters are needed in order to provide a better understanding of the effects of cognitive training.

For future studies, there are a number of additional issues that researchers must address to support empirical evidence for the implementation of EF training as a complementary intervention for individuals with ADHD. Specifically, a welldesigned intervention should (a) randomize participants into the experimental and control groups; (b) match participants in variables that might account for differences between groups other than the treatment (e.g., age, comorbidity); (c) control for participants and informants expectations through blinding and assessment of expectations prior to the beginning of the intervention to control for possible placebo effects; (d) compare the performance of the experimental group to both active and passive control groups; and (e) use both performancebased measures and ratings of EF and behavior to assess the interventions' efficacy.

Notwithstanding, this review represents an important contribution as it includes a wider range of studies (i.e., different designs and interventions), having important clinical and educational implications, as it demonstrates the feasibility and positive effects of conducting EF training with children and adolescents with ADHD in a variety of contexts.

In sum, our results showed that cognitive training can be an effective intervention for children and adolescents 
with $\mathrm{ADHD}$ and might be considered a complement of psychostimulant medication. Nonetheless, conclusions should be interpreted with caution due to important methodological limitations. However, the available evidence certainly justifies the allocation of resources to evaluate the efficacy of EF interventions, since they carry the promise of reducing ADHD symptomatology and improving academic, interpersonal, and occupational outcomes.

\section{AUTHOR CONTRIBUTIONS}

$\mathrm{AV}$ and MF contributed to the conception and design of the work. AV prepared the first draft of the manuscript.
MF revised the manuscript critically for important intellectual content. MF and SV revised the last version of the manuscript.

\section{FUNDING}

This research was supported by the M2S Project funded through the Operational Programme for Competitiveness and Internationalization, supported by FEDER and national funds allocated to the Portuguese Foundation for Science and Technology (NORTE-01-0145FEDER-028404).

\section{REFERENCES}

American Psychiatric Association (2013). Diagnostic and Statistical Manual of Mental Disorders, 5th Edn. Arlington, VA: American Psychiatric Publishing.

Anderson, P. (2002). Assessment and development of executive function (EF) during childhood. Child Neuropsychol. 8, 71-82. doi: 10.1076/chin.8.2.71.8724

Azami, S., Moghadas, A., Sohrabi-Esmrood, F., Nazifi, M., Mirmohamad, M., Hemmati, F., et al. (2016). A pilot randomized controlled trial comparing computer-assisted cognitive rehabilitation, stimulant medication, and an active control in the treatment of ADHD. Child Adolesc. Mental Health 21, 217-224. doi: $10.1111 /$ camh.12157

Barkley, R. A. (ed.). (2015). Attention-Deficit Hyperactivity Disorder: A Handbook for Diagnosis and Treatment, 4th Edn. New York, NY: Guilford Press.

Barnett, R., Maruff, P., Vance, A., Luk, E. S. L., Costin, J., Wood, C., et al. (2001). Abnormal executive function in Attention Deficit Hyperactivity Disorder: the effect of stimulant medication and age on spatial working memory. Psychol. Med. 31, 1107-1115. doi: 10.1017/S0033291701004172

Beck, S. J., Hanson, C. A., Puffenberger, S. S., Benninger, K. L., and Benninger, W. B. (2010). A controlled trial of working memory training for children and adolescents with ADHD. J. Clin. Child Adolesc. Psychol. 39, 825-836. doi: 10.1080/15374416.2010.517162

Biederman, J., and Spencer, T. J. (2008). Psychopharmacological interventions. Child Adolesc. Psychiatr. Clin. N. Am. 17, 439-458. doi: 10.1016/j.chc.2007.12.001

Bigorra, A., Garolera, M., Guijarro, S., and Hervás, A. (2016). Long-term far-transfer effects of working memory training in children with ADHD: a randomized controlled trial. Eur. Child Adolesc. Psychiatr. 25, 853-867. doi: 10.1007/s00787-015-0804-3

Boot, W. R., Simons, D. J., Stothart, C., and Stutts, C. (2013). The pervasive problem with placebos in psychology: why active control groups are not sufficient to rule out placebo effects. Perspect. Psychol. Sci. 8, 445-454. doi: $10.1177 / 1745691613491271$

Brown, T. E. (2013). A New Understanding of ADHD in Children and Adults: Executive Function Impairments. New York, NY: Routledge.

Capodieci, A., Gola, M. L., Cornoldi, C., and Re, A. M. (2018). Effects of a working memory training program in preschoolers with symptoms of attention-deficit/hyperactivity disorder. J. Clin. Exp. Neuropsychol. 40, 17-29. doi: 10.1080/13803395.2017.1307946

Cortese, S., Ferrin, M., Brandeis, D., Buitelaar, J., Daley, D., Dittmann, R. W., et al. (2015). Cognitive training for attention-deficit/hyperactivity disorder: meta-analysis of clinical and neuropsychological outcomes from randomized controlled trials. J. Am. Acad. Child Adolesc. Psychiatr. 54, 164-174. doi: 10.1016/j.jaac.2014.12.010

Davis, N. O., Bower, J., and Kollins, S. H. (2018). Proof-of-concept study of an at-home, engaging, digital intervention for pediatric ADHD. PLoS ONE 13:e0189749. doi: 10.1371/journal.pone.0189749

Dawson, P., and Guare, R. (2010). Executive Skills in Children and Adolescents: A Practical Guide to Assessment and Intervention, 2nd Edn. New York, NY: Guilford Press.
Diamond, A. (2012). Activities and programs that improve children's executive functions. Curr. Dir. Psychol. Sci. 21, 335-341. doi: 10.1177/0963721412453722

Dovis, S., Van der Oord, S., Wiers, R. W., and Prins, P. J. M. (2015). Improving executive functioning in children with ADHD: training multiple executive functions within the context of a computer game. A randomized double-blind placebo controlled trial. PLOS ONE 10, 10:e0121651. doi: 10.1371/journal.pone.0121651

Efron, D., Bryson, H., Lycett, K., and Sciberras, E. (2016). Children referred for evaluation for ADHD: comorbidity profiles and characteristics associated with a positive diagnosis. Child Care Health Dev. 42, 718-724. doi: $10.1111 /$ cch. 12364

Egeland, J., Aarlien, A. K., Saunes, B.-K., Aarlien, A. K., and Saunes, B.-K. (2013). Few effects of far transfer of working memory training in ADHD: a randomized controlled trial. PLoS ONE 8, 1-9. doi: 10.1371/journal.pone.0075660

Flook, L., Smalley, S. L., Kitil, M. J., Galla, B. M., Kaiser-Greenland, S., Locke, J., et al. (2010). Effects of mindful awareness practices on executive functions in elementary school children. J. Appl. School Psychol. 26, 70-95. doi: 10.1080/15377900903379125

Geurts, H. M., Verté, S., Oosterlaan, J., Roeyers, H., and Sergeant, J. A. (2005). ADHD subtypes: do they differ in their executive functioning profile? Arch. Clin. Neuropsychol. 20, 457-477. doi: 10.1016/j.acn.2004.11.001

Gioia, G. A., Guy, S. C., Isquith, P. K., and Kenworthy, L. (2000). Behavior Rating Inventory of Executive Function. Florida, FL: PAR.

Green, C. T., Long, D., Green, D., Iosif, A., Dixon, J., Miller, M., et al. (2012). Will working memory training generalize to improve off-task behavior in children with attention-deficit/hyperactivity disorder? Neurotherapeutics 9, 639-648. doi: 10.1007/s13311-012-0124-y

Hannesdottir, D. K., Ingvarsdottir, E., and Bjornsson, A. (2017). The OutSMARTers program for children with ADHD: a pilot study on the effects of social skills, self-regulation, and executive function training. J. Atten. Disord. 21, 353-364. doi: 10.1177/1087054713520617

Johnstone, S. J., Roodenrys, S., Blackman, R., Johnston, E., Loveday, K., Mantz, S., et al. (2012). Neurocognitive training for children with and without AD/HD. ADHD Atten. Deficit Hyperactivity Disord. 4, 11-23. doi: 10.1007/s12402-011-0069-8

Karch, D., Albers, L., Renner, G., Lichtenauer, N., and Von Kries, R. (2013). The efficacy of cognitive training programs in children and adolescents. Dtsch. Arztebl. Int. 110, 643-652. doi: 10.3238/arztebl.2013.0643

Lawrence, V., Houghton, S., Douglas, G., Durkin, K., Whiting, K., and Tannock, R. (2004). Executive function and ADHD: a comparison of children's performance during neuropsychological testing and real-world activities. J. Atten. Disord. 7, 137-149. doi: 10.1177/108705470400700302

McGuiness, L. A. (2019). robvis: An R Package and Web Application for Visualizing Risk-of-Bias Assessments. Retrieved from: https://github.com/mcguinlu/robvis (accessed December 13, 2019).

Menezes, A., Dias, N. M., Trevisan, B. T., Carreiro, L. R. R., Seabra, A. G., and Gotuzo, R. A. (2015). Intervention for executive functions in attention deficit and hyperactivity disorder. Arq. Neuro Psiquiatr. 73, 227-236. doi: 10.1590/0004-282X20140225 
Minder, F., Zuberer, A., Eis, D., Drechsler, R., Brandeis, D., and Drechsler, R. (2018). Informant-related effects of neurofeedback and cognitive training in children with ADHD including a waiting control phase: a randomized-controlled trial. Eur. Child Adolesc. Psychiatr. 27, 1055-1066. doi: 10.1007/s00787-018-1116-1

Moher, D., Liberati, A., Tetzlaff, J., Altman, D. G., and The PRISMA Group. (2009). Preferred reporting items for systematic reviews and meta-analyses: The PRISMA Statement. Ann. Int. Med. 151, 264-270. doi: 10.7326/0003-4819-151-4-200908180-00135

Nigg, J. T. (2006). What Causes ADHD? Understanding What Goes Wrong and Why. New York, NY: The Guilford Press.

Nigg, J. T., Blaskey, L. G., Huang-Pollock, C. L., and Rappley, M. D. (2002). Neuropsychological executive functions and DSM-IV ADHD subtypes. J. Am. Acad. Child Adolesc. Psychiatr. 41, 59-66. doi: 10.1097/00004583-200201000-00012

Ouzzani, M., Hammady, H., Fedorowicz, Z., and Elmagarmid, A. (2016). Rayyan-a web and mobile app for systematic reviews. Syst. Rev. 5:210. doi: 10.1186/s13643-016-0384-4

Pasini, A., Paloscia, C., Alessandrelli, R., Porfirio, M. C., and Curatolo, P. (2007). Attention and executive functions profile in drug naive ADHD subtypes. Brain Dev. 29, 400-408. doi: 10.1016/j.braindev.2006.11.010

Qian, Y., Chen, M., Shuai, L., Cao, Q.-J., Yang, L., and Wang, Y.-F. (2017). Effect of an ecological executive skill training program for school-aged children with Attention Deficit Hyperactivity Disorder: a randomized controlled clinical trial. Chin. Med. J. 130, 1513-1522. doi: 10.4103/0366-6999.208236

Rabipour, S., and Raz, A. (2012). Training the brain: fact and fad in cognitive and behavioral remediation. Brain Cogn. 79, 159-179. doi: 10.1016/j.bandc.2012.02.006

Rapport, M. D., Chung, K.-M., Shore, G., and Isaacs, P. (2001). A conceptual model of child psychopathology: implications for understanding Attention Deficit Hyperactivity Disorder and treatment efficacy. J. Clin. Child Adolesc. Psychol. 30, 48-58. doi: 10.1207/S15374424JCCP3001_6

Rapport, M. D., Orban, S. A., Kofler, M. J., and Friedman, L. M. (2013). Do programs designed to train working memory, other executive functions, and attention benefit children with ADHD? A meta-analytic review of cognitive, academic, and behavioral outcomes. Clin. Psychol. Rev. 33, 1237-1252. doi: 10.1016/j.cpr.2013.08.005

Re, A. M., Capodieci, A., and Cornoldi, C. (2015). Effect of training focused on executive functions (attention, inhibition, and working memory) in preschoolers exhibiting ADHD symptoms. Front. Psychol. 6:1161. doi: 10.3389/fpsyg.2015.01161

Reale, L., Bartoli, B., Cartabia, M., Zanetti, M., Costantino, M. A., Canevini, M. P., et al. (2017). Comorbidity prevalence and treatment outcome in children and adolescents with ADHD. Eur. Child Adolesc. Psychiatr. 26, 1443-1457. doi: 10.1007/s00787-017-1005-Z

Roberts, W., Milich, R., and Barkley, R. A. (2015). "Primary symptoms, diagnostic criteria, subtyping, and prevalence of ADHD," in Attention-Deficit Hyperactivity Disorder: A Handbook for Diagnosis and Treatment, 4th Edn. eds R. A. Barkley (New York, NY: Guilford Press), 51-80.

Shuai, L., Daley, D., Wang, Y.-F., Zhang, J.-S., Kong, Y.-T., Tan, X., et al. (2017). Executive function training for children with Attention Deficit Hyperactivity Disorder. Chin. Med. J. 130:549. doi: 10.4103/0366-6999.200541

Sonuga-Barke, E. J. S. (2003). The dual pathway model of AD/HD: an elaboration of neuro-developmental characteristics. Neurosci. Biobehav. Rev. 27, 593-604. doi: 10.1016/j.neubiorev.2003.08.005

Sonuga-Barke, E. J. S., Brandeis, D., Cortese, S., Daley, D., Ferrin, M., Holtmann, M., et al. (2013). Nonpharmacological interventions for ADHD: systematic review and meta-analyses of randomized controlled trials of dietary and psychological treatments. Am. J. Psychiatr. 170, 275-289. doi: 10.1176/appi.ajp.2012.12070991

Steiner, N. J., Frenette, E. C., Rene, K. M., Brennan, R. T., and Perrin, E. C. (2014). Neurofeedback and cognitive attention training for children with attentiondeficit hyperactivity disorder in schools. J. Dev. Behav. Pediatr. 35, 18-27. doi: 10.1097/DBP.0000000000000009

Steiner, N. J., Sheldrick, R. C., Gotthelf, D., and Perrin, E. C. (2011). Computer-based attention training in the schools for children with Attention Deficit/Hyperactivity Disorder: a preliminary trial. Clin. Pediatr. 50, 615-622. doi: 10.1177/0009922810397887

Sterne, J. A., Hernán, M. A., Reeves, B. C., Savovi,ć, J., Berkman, N. D., Viswanathan, M., et al. (2016). ROBINS-I: a tool for assessing risk of bias in non-randomised studies of interventions. BMJ 355:i4919. doi: 10.1136/bmj.i4919

Sterne, J. A. C., Savović, J., Page, M. J., Elbers, R. G., Blencowe, N. S., Boutron, I., et al. (2019). RoB 2: a revised tool for assessing risk of bias in randomised trials. BMJ 366:14898. doi: 10.1136/bmj. 14898

Swanson, J., Baler, R. D., and Volkow, N. D. (2011). Understanding the effects of stimulant medications on cognition in individuals with attention-deficit hyperactivity disorder: a decade of progress. Neuropsychopharmacology 36, 207-226. doi: 10.1038/npp.2010.160

Tamm, L., Epstein, J. N., Peugh, J. L., Nakonezny, P. A., and Hughes, C. W. (2013). Preliminary data suggesting the efficacy of attention training for school-aged children with ADHD. Dev. Cogn. Neurosci. 4, 16-28. doi: 10.1016/j.den.2012.11.004

Tamm, L., and Nakonezny, P. A. (2015). Metacognitive executive function training for young children with ADHD: a proof-of-concept study. ADHD Atten. Deficit Hyperactivity Disord. 7, 183-190. doi: 10.1007/s12402-014-0162-x

Toplak, M. E., Connors, L., Shuster, J., Knezevic, B., and Parks, S. (2008). Review of cognitive, cognitive-behavioral, and neural-based interventions for attention-deficit/hyperactivity disorder (ADHD). Clin. Psychol. Rev. 28, 801-823. doi: 10.1016/j.cpr.2007.10.008

Trani, M., Di, Casini, M. P., Capuzzo, F., Gentile, S., Bianco, G., Menghini, D., et al. (2011). Executive and intellectual functions in attentiondeficit/hyperactivity disorder with and without comorbidity. Brain Dev. 33, 462-469. doi: 10.1016/j.braindev.2010.06.002

van der Donk, M., Hiemstra-Beernink, A.-C., Tjeenk-Kalff, A., van der Leij, A., and Lindauer, R. (2015). Cognitive training for children with ADHD: a randomized controlled trial of Cogmed working memory training and 'paying attention in class'. Front. Psychol. 6:1081. doi: 10.3389/fpsyg.2015. 01081

van der Oord, S., Ponsioen, A. J., Geurts, H. M., Ten Brink, E. L., Prins, P. J. (2014). A pilot study of the efficacy of a computerized executive functioning remediation training with game elements for children with $\mathrm{ADHD}$ in an outpatient setting: outcome on parent- and teacher-rated executive functioning and ADHD behavior. J. Attent. Disord. 18, 699-712. doi: $10.1177 / 1087054712453167$

van Dongen-Boomsma, M., Vollebregt, M. A., Buitelaar, J. K., and Slaats-Willemse, D. (2014). Working memory training in young children with ADHD: a randomized placebo-controlled trial. J. Child Psychol. Psychiatr. 55, 886-896. doi: $10.1111 /$ jcpp. 12218

Vinogradov, S., Fisher, M., and de Villers-Sidani, E. (2012). Cognitive training for impaired neural systems in neuropsychiatric illness. Neuropsychopharmacology 37, 43-76. doi: 10.1038/npp.2011.251

Weyandt, L. L., and Gudmundsdottir, B. G. (2015). "Developmental and neuropsychological deficits in children with ADHD," in Attention-Deficit Hyperactivity Disorder: A Handbook for Diagnosis and Treatment, 4th Edn. ed R. A. Barkley (New York, NY: Guilford Press), 116-139.

Willcutt, E. G., Doyle, A. E., Nigg, J. T., Faraone, S. V., and Pennington, B. F. (2005). Validity of the executive function theory of attentiondeficit/hyperactivity disorder: a meta-analytic review. Biol. Psychiatr. 57, 1336-1346. doi: 10.1016/j.biopsych.2005.02.006

Yáñez-Téllez, G., Romero-Romero, H., Rivera-García, L., Prieto-Corona, B., Bernal-Hernández, J., Marosi-Holczberger, E., et al. (2012). Cognitive and executive functions in ADHD. Actas Esp. Psiquiatr. 40, 293-298.

Yildiz, O., Sismanlar, S. G., Memik, N. C., Karakaya, I., and Agaoglu, B. (2011). Atomoxetine and methylphenidate treatment in children with ADHD: the efficacy, tolerability and effects on executive functions. Child Psychiatr. Hum. Dev. 42, 257-269. doi: 10.1007/s10578-010-0212-3

Conflict of Interest: The authors declare that the research was conducted in the absence of any commercial or financial relationships that could be construed as a potential conflict of interest.

Copyright (c) 2020 Veloso, Vicente and Filipe. This is an open-access article distributed under the terms of the Creative Commons Attribution License (CC BY). The use, distribution or reproduction in other forums is permitted, provided the original author(s) and the copyright owner(s) are credited and that the original publication in this journal is cited, in accordance with accepted academic practice. No use, distribution or reproduction is permitted which does not comply with these terms. 